\title{
The external shear in the gravitationally lensed system Q 2237+0305: A two-plane lens modelling
}

\author{
B. M. Mihov
}

\author{
Institute of Astronomy, Bulgarian Academy of Sciences and Isaac Newton Institute of Chile, Bulgarian Branch \\ 72 Tsarigradsko Chausse Blvd., 1784 Sofia, Bulgaria \\ Received 13 July 1998 / Accepted 2 February 2001
}

\begin{abstract}
We present one-plane lens models (with and without an external shear added) and a two-plane lens model for the gravitationally lensed system Q $2237+0305$. The first (the main) lens plane is at $z=0.0394$ and the second lens plane is at $z=0.5664$. We found the best lens solutions for this system up to now $-\chi_{\mathrm{df}}^{2}=0.35$ for the external shear model and $\chi_{\mathrm{df}}^{2}=0.44$ for the two-plane model. Generally, we found a nearly singular (with an upper limit on the core radius of $\approx 36 \mathrm{pc}$ ) pseudoisothermal mass distribution for the central part of the main lens that is consistent with the available observations of the Q $2237+0305$ system (except the misalignment of about $13^{\circ}$ between the mass and the light distributions). Furthermore, the main source of the external shear is possibly the object connected with the Mg II absorption. In any case the external perturbations should be taken into account in the future models of the gravitationally lensed system Q 2237+0305. For the two-plane lens model the blue mass-to-light ratio for the central $1^{\prime \prime}$ of the $\mathrm{SBb}$ galaxy-lens is estimated to be $6.8_{-0.1}^{+0.3} \mathcal{M}_{\odot} / \mathcal{L}_{\odot, B}$, and the mass of the SBb galaxy inside the mean ring of the images is $\mathcal{M}\left(\leqq 0^{\prime \prime} 885\right)=1.471_{-0.010}^{+0.069} 10^{10} \mathcal{M} \odot$. The introduction of the second lens plane leaves the core radius, the axis ratio and the position angle of the mass distribution in the main lens almost unchanged but decreases the mass inside the mean ring of the images with $1.2 \%$ and increases the total magnification of the images by a factor of $\approx 1.5$. The parameters of the mass distribution in the second lens are not very well constrained. If the second lens is a single galaxy we set a lower limit on the radius of the $\mathrm{Mg}$ II absorbing halo of $\approx 14 \mathrm{kpc}$. The probability for the two-plane lens model is estimated to be $8.010^{-9}$.
\end{abstract}

Key words. quasars: absorption lines - quasars: individual: Q 2237+0305 - gravitational lensing

\section{Introduction}

The gravitationally lensed system Q 2237+0305 (hereafter GLS Q 2237+0305) was found accidentally by Huchra et al. (1985) during the Center for Astrophysics Redshift Survey and looks like a cross - the four images of the flatspectrum radio-quiet quasar Q $2237+0305(z=1.695)$ are situated nearly symmetrically around the bright nucleus of the nearby $(z=0.0394) \mathrm{SBb}$ galaxy $2237+0305$ (Zwicky et al. 1965; Huchra et al. 1985; Yee 1988; Falco et al. 1996). According to Huchra et al. (1985) the lensing galaxy $2237+0305$ is located in the outskirts of Pegasus II cluster of galaxies. One moderate redshift $(z=0.5664)$ and three high redshift $(z=0.9674, z=0.9709$ and $z=0.9713) \mathrm{Mg}$ II absorption line systems have been detected in the spectrum of the lensed quasar Q 2237+0305 (Hintzen et al. 1990; Foltz et al. 1992). Mediavilla et al. (1998) detected an arc of extended emission (C III]) connecting the $\mathrm{A}, \mathrm{B}$ and $\mathrm{D}$ quasar images.

Keeton et al. (1997) and Witt \& Mao (1997) pointed out the need of a second, independent, external shear axis

Send offprint requests to: B. M. Mihov,

e-mail: bmihov@astro.bas.bg (in addition to the main lens ellipticity) in order to get an acceptable fit to the observations of the gravitationally lensed systems. This external shear could be produced by a mass distribution correlated with the main lens and/or by massive objects along the line of sight ${ }^{1}$. In the case of the GLS Q 2237+0305 there are three possible sources of an external shear perturbation that could contribute to the lensing of the quasar: the galaxy bar (Yee 1988; Kent \& Falco 1988; Schmidt et al. 1998), the mass connected with Pegasus II galaxy cluster (Webster et al. 1991) and the mass associated with the Mg II absorption (Kochanek \& Apostolakis 1988; Webster et al. 1991; Foltz et al. 1992; Rix et al. 1992). So, it is quite reasonable to try a lens solution with the external perturbation taken into account. Another reason to try more complicated models is the failure of the one-component models published so far to fit satisfactorily the observations of the GLS Q 2237+0305. Moreover, the explicit modelling of the perturbers

\footnotetext{
1 Another source of an independent shear could be the misalignment between the mass and the light distribution (Keeton et al. 1997) but we shall not consider this possibility in the paper (see Sect. 3.2; see also Keeton et al. 1998).
} 
provides for us the parameters of their mass distribution (e.g. Schmidt et al. 1998).

The GLS Q 2237+0305 has been modelled many times (Huchra et al. 1985; Schneider et al. 1988; Kent \& Falco 1988; Kochanek 1991; Minakov \& Shalyapin 1991; Rix et al. 1992; Wambsganss \& Paczyński 1994; Witt et al. 1995; Williams \& Saha 1995; Petrov et al. 1996; Falco et al. 1996; Schmidt et al. 1998; Chae et al. 1998; Mediavilla et al. 1998; see also Kassiola \& Kovner 1995; Witt 1996; Witt \& Mao 1997) but only Witt \& Mao (1997) and Schmidt et al. (1998) made an attempt to include a second, independent shear axis in the models of this lensed system. Witt \& Mao (1997) used a general analytical approach and found a minimal shear required to fit the observed positions of $0.0099 \pm 0.0063$ (see also Witt 1996). Schmidt et al. (1998) assumed that the source of the external shear is the galaxy bar. Modelling the galaxy mass distribution as a singular power-law ellipsoid aligned with the disk+bulge light distribution and the bar - as a Ferrers bar (and fitting only the positions) they found quite a good fit $-\chi_{\min }^{2} / 3=1.3-3.4$, and were able to derive the mass of the bar.

In the framework of the above discussion we put the following problems to be solved in this paper. The first problem is to find a general external shear solution for the GLS Q 2237+0305. The next problems are to model the GLS Q $2237+0305$ by means of a two-plane lens taking explicitly into account the lensing effect of the object associated with the Mg II absorption and to extract some information about the second lens. Finally, we use the most accurate (up to now) ultraviolet image positions combined with realistic radio flux ratios of the images as observational constraints on our models.

In order to solve the above problems we construct a one-plane model with an external shear added and a twoplane model. One-plane models without an external shear are added in order to be compared with more complicated models. In the two-plane model the first (the main) lens plane is at $z=0.0394$ and the second lens plane is at $z=0.5664$, whereas the one-plane models include only the main lens plane. We are forced to use only two planes in order to build a well constrained detailed lens model (the use of multiple lens plane approach increases the number of the free parameters). We choose the second plane to be at $z=0.5664$ not at $z \approx 0.97$ because of the fact that an object at $z=0.5664$ has larger convergence and therefore, it has stronger lensing effect than at $z \approx 0.97$.

The paper is organized as follows. In Sect. 2 we describe the observational constraints, the lens mass models and the modelling procedure used. The results from the computations are presented and discussed in Sect. 3. Finally, in Sect. 4 we summarize the findings in this paper and discuss some aspects of the future observations and modelling of the GLS Q 2237+0305.

Throughout the paper we use the angular diameter distances $D_{i j} \equiv D\left(z_{i}, z_{j}\right)$ given in Blandford \& Narayan (1992) and Einstein - de Sitter $\left(\Omega_{0}=1, \Lambda_{0}=0\right)$ Universe with Hubble parameter $H_{0}=75 \mathrm{~km} \mathrm{~s}^{-1} \mathrm{Mpc}^{-1}$.
Table 1. Observed ultraviolet positions of the images and of the $\mathrm{SBb}$ galaxy and radio flux ratios of the images

\begin{tabular}{lrcc}
\hline$i$ & \multicolumn{1}{c}{$\theta_{i, 1}[\operatorname{arcsec}]$} & $\theta_{i, 2}[\operatorname{arcsec}]$ & $\mu_{i \mathrm{~A}}^{\text {obs }}$ \\
\hline $\mathrm{A}$ & $0.0000 \pm 0.0015$ & $0.0000 \pm 0.0015$ & 1.00 \\
$\mathrm{~B}$ & $0.6710 \pm 0.0015$ & $1.6970 \pm 0.0015$ & $1.08 \pm 0.27$ \\
$\mathrm{C}$ & $-0.6340 \pm 0.0015$ & $1.2100 \pm 0.0015$ & $0.55 \pm 0.21$ \\
$\mathrm{D}$ & $0.8660 \pm 0.0015$ & $0.5280 \pm 0.0015$ & $0.77 \pm 0.23$ \\
$\mathrm{G}$ & $0.0810 \pm 0.0100$ & $0.9370 \pm 0.0100$ & - \\
\hline
\end{tabular}

\section{Observational constraints, lens mass models and modelling procedure}

\subsection{Observational constraints}

We use the coordinates ${ }^{2}$ and the flux ratios of the quasar images relative to the A quasar image (taken as a reference image) as observational constraints on the model free parameters. So, we have a total of 9 observational constraints.

Among the published astrometry for the GLS Q 2237+0305 (Yee 1988; Irwin et al. 1989; Crane et al. 1991; Racine 1991; Rix et al. 1992; Teuber et al. 1994; Østensen et al. 1996; Falco et al. 1996; Vakulik et al. 1997; Burud et al. 1998; Blanton et al. 1998), the most accurate image positions (and less affected by systematical errors) are those derived by Blanton et al. (1998) with the postrepaired Hubble Space Telescope (HST) in the ultraviolet band. We shall use these image positions (listed in Table 1) as observational constraints on the macromodels. Using the image and the SBb galaxy positions we derive the radius of the mean ring formed by the images to be 0. ". 885 $(=631.5 \mathrm{pc})$.

Racine (1991) claims a discovery of the fifth (E) quasar image in the GLS Q $2237+0305$ at a position $\boldsymbol{\theta}_{\mathrm{E}}^{\text {obs }}=$ $\left(0^{\prime \prime} 038,0\right.$. . 881$)$ but this discovery has not been confirmed yet (Crane et al. 1991; Fitte \& Adam 1994; Falco et al. 1996; Blanton et al. 1998).

According to Falco et al. (1996) (see also Witt et al. 1995) the radio observations are more likely to determine the real flux ratios. So, we assume that despite their low accuracy the radio flux ratios determined by Falco et al. (1996) at $\lambda=3.6 \mathrm{~cm}$ with the Very Large Array (VLA) are very close to the real ratios and we shall use them as observational constraints on the macromodels (Table 1 ).

Furthermore, according to Falco et al. (1996) the fifth quasar image should have a flux density less than one fifth of the A image flux density, i.e. $\mu_{\mathrm{EA}}^{\mathrm{obs}}<0.2$.

\footnotetext{
2 Throughout the paper we use a Cartesian coordinate system with an origin centered on the A quasar image, $\theta_{1}$-axis increases to the right (West), $\theta_{2}$-axis increases to the top (North). We also use a polar coordinate system $(\theta, \varphi)$ centered on the $\mathrm{SBb}$ galaxy with axes that point toward West $\left(\theta_{1}\right)$ and North $\left(\theta_{2}\right)$; the polar angle $\varphi$ is measured in a right handed fashion from the positive $\theta_{2}$-axis (i.e. North of West; $0^{\circ} \leqq \varphi \leqq 180^{\circ}$ ). Then the position angle PA (East of North) is simply $\mathrm{PA}=\varphi-90^{\circ}$.
} 


\subsection{Lens mass models}

We investigate three classes of lens models for the GLS Q $2237+0305$ based on the isothermal mass distribution.

The standard (one-plane one-component) class of lens models contains a SIE model (a singular isothermal elliptical mass distribution model) and a PIE model (a pseudoisothermal elliptical mass distribution model). The shear class of lens models contains a PIE $+\mathrm{XS}$ model (a PIE model with an external shear added). For both classes of lens models the lens plane is at $z=0.0394$ and includes the $\mathrm{SBb}$ galaxy (referred to as the main lens).

The two-plane class of lens models contains a PIE+SIS model (a PIE model for the main lens and a singular isothermal spherical mass distribution model for the second lens). The first (the main) lens plane is at $z=0.0394$ and includes the SBb galaxy, and the second lens plane is at $z=0.5664$ and includes the object associated with the Mg II absorption.

The convergence ${ }^{3}$ of the pseudoisothermal elliptical mass distribution (PIE, Kassiola \& Kovner 1993) has the following form:

$k(\boldsymbol{\theta})=k(0)\left[1+\frac{\theta_{1}^{2}}{\varpi^{2}(1+\varepsilon)^{2}}+\frac{\theta_{2}^{2}}{\varpi^{2}(1-\varepsilon)^{2}}\right]^{-1 / 2}$,

where $k(0)$ is the central convergence of the lens. The parameters which characterize the PIE model are the model velocity dispersion $\sigma_{\mathrm{m}}\left(k(0) \sim \sigma_{\mathrm{m}}^{2} / \varpi\right)$, the core radius $\varpi$, the elliptical parameter $\varepsilon(0 \leqq \varepsilon<1)$, the polar angle $\varphi_{\varepsilon}$ of the major axis and the position $\boldsymbol{\theta}_{0}$. The elliptical parameter is connected with the minor-to-major axis ratio $f$ of the lens mass distribution via the relationship $f=(1-\varepsilon) /(1+\varepsilon)$. In the case of the SIE model we set $\varpi=0$ and for the PIE+SIS model we set $\varpi=\varepsilon=0$ for the second lens.

The lensing potential of the external shear perturbation reads

$\phi(\theta, \varphi)=\frac{\gamma}{2} \theta^{2} \cos 2\left(\varphi-\varphi_{\gamma}\right)$,

where $\gamma$ is the shear magnitude and $\varphi_{\gamma}$ is the shear orientation. In the framework of the multipole-Taylor expansion (Trotter et al. 2000) the angle $\varphi_{\gamma}$ (and $\varphi_{\gamma}+\pi$ ) is the angle to the mass deficit, whereas the angle $\varphi_{\Sigma}=\varphi_{\gamma}+\pi / 2$ (and $\left.\varphi_{\Sigma}+\pi\right)$ is the angle to the mass excess.

For the two-plane PIE+SIS model the lens equations are taken from Erdl \& Schneider's (1993) paper. The second lens is described by its model velosity dispersion $\sigma_{\mathrm{m}}^{(2)}$ and by its position $\boldsymbol{\theta}_{0}^{(2)}$.

In all models we suppose that the centre of the main lens mass distribution coincides with the centre of brightness of the SBb galaxy, i.e. $\boldsymbol{\theta}_{0} \equiv \boldsymbol{\theta}_{\mathrm{G}}=\left(0^{\prime \prime} 081,0^{\prime \prime} \cdot 937\right)$ (see Table 1), in order to avoid the use of the main lens coordinates as free parameters.

${ }^{3}$ The convergence is the surface mass density $\Sigma$ in units of the critical one $\Sigma_{\text {crit }}$. In the case of the GLS Q $2237+0305$ we have $\Sigma_{\text {crit }}=2.482 \mathrm{~g} \mathrm{~cm}^{-1}$.

\subsection{Modelling procedure}

We use the backward ray-shooting algorithm proposed by Kayser (1990) for the lens equation inversion. As a $\chi^{2}$ function we use Kochanek's (1991) $\chi_{T}^{2}$ function modified by us to include the flux ratios, to take into account the variances of the observational constraints (see Table 1) and to use the source position corresponding to the A image as a model source position. So, our $\chi^{2}$ function has the following form:

$\chi^{2}=\sum_{i} \chi_{\theta, i}^{2}+\sum_{i} \chi_{\mu, i}^{2}$

where

$\chi_{\theta, i}^{2}=\left[\frac{\boldsymbol{\mu}_{i} \cdot\left(\boldsymbol{\beta}_{i}-\boldsymbol{\beta}_{\mathrm{A}}\right)}{\sigma_{\theta, i}}\right]^{2}$,

$\chi_{\mu, i}^{2}=\left[\frac{\mu_{i \mathrm{~A}}^{\mathrm{obs}}-\left|\mu_{i \mathrm{~A}}^{\mathrm{calc}}\right|}{\sigma_{\mu, i}}\right]^{2}$,

$i=\mathrm{B}, \mathrm{C}, \mathrm{D}, \sigma_{\theta, i}$ and $\sigma_{\mu, i}$ are the standard deviations of the position and of the flux ratio of the $i$ th image, respectively, $\boldsymbol{\mu}_{i}$ is the magnification tensor at the position of the $i$ th image, $\boldsymbol{\beta}_{i}$ is the model source position corresponding to the $i$ th image and $\mu_{i \mathrm{~A}}$ is the flux ratio (observed and calculated) of the $i$ th image relative to the A image. In order to minimize this $\chi^{2}$ function we use a downhill simplex method (Press et al. 1986 - their routine "amoeba"). We estimate the quality of the fit making use of the $\chi^{2}$ value at the minimum $\left(\chi_{\min }^{2}\right)$ divided by the degrees of freedom $\left(n_{\mathrm{df}}=N_{\text {constraints }}-N_{\text {parameters }}\right)$ for the particular model, i.e. $\chi_{\mathrm{df}}^{2}=\chi_{\min }^{2} / n_{\mathrm{df}}$.

The finding of the global minimum in the multidimensional $\chi^{2}$ surface is an arduous task. In general, the multidimensional $\chi^{2}$ surface has many local minima together with the global one and the minimization schemes frequently converge on local minima. However, there are cases (simple models with few (2-3) free parameters) when the minimization programme converges stably on the global minimum. We use the following technique in order to find the global minimum of the $\chi^{2}$ function. We must choose an initial simplex in the parameter space in order to work with the downhill simplex method. When there is a single minimum the final result is independent of the initial simplex choice and we have no problems. When there are many minima in the $\chi^{2}$ surface, the final result will depend on the initial simplex choice, i.e. the programme will converge on different minima with different initial conditions. The more different initial simplexes we use, the bigger the probability to find the global minimum gets in the latter case. Clearly, for different problems a different number of cycles will be required before we can have confidence in the results. Applying this technique, we define an appropriate interval for each parameter in the begining of the modelling. In each minimization cycle we choose the vertices of the initial simplex randomly in these intervals and then minimize $\chi^{2}$. After some number of minimization cycles we could change the parameters' 
intervals. At the end of each cycle we write the final result in a data file and then we use these data in the determination of the joint confidence region for all $n$ parameters of the model - we find all successful models which increase the $\chi^{2}$ function up to the desirable confidence level $P \%$ (i.e. we find all models with $\chi_{n, P \%}^{2}=\chi_{\min }^{2}+\Delta \chi_{n, P \%}^{2}$ ) and we choose the most deviant parameters of these models as confidence limits on the best model parameters. When we have a well defined minimum it is possible to use the standard procedure to find the confidence limits instead of the data file generated, i.e. we vary the parameter of interest until we get $\chi_{1, P \%}^{2}=\chi_{\min }^{2}+\Delta \chi_{1, P \%}^{2}$ while the remaining parameters are adjusted to minimize $\chi^{2}$ (some values of $\Delta \chi_{n, P \%}^{2}$ are given in Press et al. 1986). Each minimum we suspect to be the global one is checked via the squared deviation function (SDF) method (Schramm \& Kayser 1987; Kayser \& Schramm 1988) in order to search for extra images produced by the model; these images could be real but too faint to be detected or could be unreal ones. Recently, Keeton et al. (2000) showed that in the presence of a strong tidal perturbation oriented near the lens minor axis six or eight highly magnified images could be produced but this is not our case (see Table 2); in fact, we could not find extra images having brightness comparible to or smaller than the brightness of the observed images. In order to draw the caustics and the critical curves we find the points where the Jacobian of the mapping "image plane $\rightarrow$ source plane" changes its sign. As the mass model of the main lens is nonsingular the central (fifth, E) image will be created. In order to predict the position and the flux ratio of the E quasar image we use the following approach. We cover the central part of the modelled region (where the fifth E image is expected to be) with an equally spaced two dimensional grid. Each grid point is then traced back to the source plane via the gravitational lens equation and the corresponding source position is found. The grid point corresponding to the source point having smallest distance to the derived source position is selected. A new and more closely spaced grid is then constructed in the outskirts of the selected point and the entire procedure is repeated until the desired accuracy is achieved. After that the magnification of the E image is computed. Finally, in order to derive the lens mass inside a given radius we integrate the lens convergence within the selected radius.

\section{Results and discussion}

We first calculate two standard (one-plane onecomponent) lens models. In this way we can follow the changes in the fit quality and in the parameters of the main lens mass distribution due to the use of a shear or of a two-plane instead of a standard lens model. We can also test the standard models using the highest accuracy image positions ever used.

In the shear models we assume that the standard model is perturbed by the mass distribution correlated with the main lens and/or by massive objects along the line of sight.

In the two-plane model we assume that the object connected with the Mg II absorption has nonvanishing lensing effect thereby it influences the formation of the images.

We found the best lens solution for the GLS Q $2237+0305$ up to now - the PIE+XS and the PIE+SIS models with $\chi_{\mathrm{df}}^{2}=0.35$ and $\chi_{\mathrm{df}}^{2}=0.44$, respectively. The results from the computations are summarized in Table 2. The errors quoted are $68.3 \%$ confidence limits on the parameters. The error of the mass accounts only for the error of the model velocity dispersion. In Table 3 we list the terms $\left(\chi_{\theta, i}^{2}, \chi_{\mu, i}^{2}\right)$ (Eqs. (4), (5)) for each image in order to get an overview on the ability the given model to fit the observations. The observed and predicted image positions and magnifications by the PIE $+\mathrm{XS}$ model are plotted in Fig. 1, and the caustics and the critical curves for the PIE+SIS model are plotted in Fig. 2 along with the direction to the mass excess found by the PIE + XS model.

The position and flux ratio of the fifth quasar image predicted by the PIE model are $\left(\boldsymbol{\theta}_{\mathrm{E}}^{\text {calc }} ; \mu_{\mathrm{EA}}^{\text {calc }}\right)=$ $(0 \prime$. 032,0 , $950 ; 0.11)$. The PIE+XS and the PIE+SIS models are nearly singular and the fifth image is strongly demagnified. For the PIE+XS model we found the mass excess angle to be $\varphi_{\Sigma}=20.01_{-19.80}^{+13.50}$. The polar coordinates of the second lens relative to the main lens are $\left(\theta^{(2)}, \varphi^{(2)}\right)=\left(2^{\prime \prime} .704,15.76\right)$ (PIE+SIS model). Before calculating the polar coordinates we made the transformation $\boldsymbol{\theta}_{0}^{(2)}=\boldsymbol{\theta}_{0}^{(2)}-\boldsymbol{\theta}_{0}$, where $\boldsymbol{\theta}_{0}$ is the position of the $\mathrm{SBb}$ galaxy relative to the $\mathrm{A}$ image. The polar coordinates of Pegasus II cluster relative to the main lens are $\left(\theta^{(\mathrm{PII})}, \varphi^{(\mathrm{PII})}\right) \approx\left(9^{\circ}, 150^{\circ}\right)$ (Chincarini \& Rood 1976).

Finally, we found the $B$ magnitudes of the unlensed quasar to be 19.69 and 20.15 for the PIE+XS and the PIE+SIS models, respectively. We have used the $B$ magnitudes (the PSF fitting method) of the images derived by Burud et al. (1998) - the total $B$ magnitude of the lensed quasar is 16.66 .

\subsection{Fit quality}

The common feature of the standard models published so far is their failure in the fitting of the observations - typically one has $\chi_{\mathrm{df}}^{2}>2$. Our models are not an exception (Table 2). Our worst model - the SIE model (Table 3), leads to differences between the observed and the calculated image positions that are up to $\approx 10$ times the standard deviations of the observed image positions. The flux ratios for this model are fitted within their observational errors but the last ones are too large to say that the ratios are fitted well (this is true for all other models computed in the paper except for the D image in the PIE model). One can see that the introduction of the core radius in the PIE model leads to a better fit of the positions and to a worse fit of the flux ratios compared to the singular SIE model - in particular, the flux ratio of the D image is fitted rather badly despite the large observational 
Table 2. The final $\chi_{\mathrm{df}}^{2}$ (row 1; in the parenthesis we show the number of degrees of freedom), the parameters (rows 2-10), the calculated source position (row 11; it corresponds to the A image source position), the total magnification (row 12) and the mass of the main lens inside the mean ring of the images (row 13) for the computed models

\begin{tabular}{|c|c|c|c|c|c|}
\hline Parameter & SIE & PIE & $\mathrm{PIE}+\mathrm{XS}$ & $\mathrm{PIE}+\mathrm{SIS}$ & Unit \\
\hline$\chi_{\mathrm{df}}^{2}\left(n_{\mathrm{df}}\right)$ & $36.54(6)$ & $8.28(5)$ & $0.35(3)$ & $0.44(2)$ & - \\
\hline$\sigma_{\mathrm{m}}$ & $182.8_{-0.1}^{+0.1}$ & $220.0_{-2.6}^{+2.6}$ & $182.3_{-0.4}^{+2.2}$ & $181.1_{-0.6}^{+4.2}$ & $\mathrm{~km} \mathrm{~s}^{-1}$ \\
\hline$\varpi$ & $\ldots$ & $0.364_{-0.023}^{+0.023}$ & $310^{-7+0.02}$ & $310^{-5}+0.05$ & $\operatorname{arcsec}$ \\
\hline$\varepsilon$ & $0.209_{-0.002}^{+0.002}$ & $0.079_{-0.005}^{+0.005}$ & $0.195_{-0.030}^{+0.018}$ & $0.191_{-0.036}^{+0.013}$ & - \\
\hline$\varphi_{\varepsilon}$ & $157.55_{-0.04}^{+0.05}$ & $157.32_{-0.06}^{+0.06}$ & $154.34_{-0.47}^{+0.74}$ & $154.31_{-0.06}^{+0.76}$ & degree \\
\hline$\gamma$ & $\ldots$ & $\ldots$ & $0.012_{-0.001}^{+0.005}$ & $\ldots$ & - \\
\hline$\varphi_{\gamma}$ & $\cdots$ & $\ldots$ & $110.01_{-19.80}^{+13.50}$ & $\ldots$ & degree \\
\hline$\sigma_{\mathrm{m}}^{(2)}$ & $\ldots$ & $\ldots$ & $\ldots$ & $253.6_{-80.4}^{+29.2}$ & $\mathrm{~km} \mathrm{~s}^{-1}$ \\
\hline$\theta_{0,1}^{(2)}$ & $\ldots$ & $\ldots$ & $\ldots$ & $2.684_{-5.586}^{+0.652}$ & $\operatorname{arcsec}$ \\
\hline$\theta_{0,2}^{(2)}$ & .. & $\ldots$ & $\ldots$ & $1.671_{-0.839}^{+0.947}$ & $\operatorname{arcsec}$ \\
\hline$\left(\beta_{1}, \beta_{2}\right)$ & $(0.145,0.927)$ & $(0.114,0.930)$ & $(0.142,0.927)$ & $(1.005,1.180)$ & $\operatorname{arcsec}$ \\
\hline$\mu_{\text {tot }}$ & 15.43 & 52.44 & 16.27 & 24.97 & - \\
\hline $\mathcal{M}\left(\leqq 0^{\prime \prime} 885\right)$ & $1.490_{-0.020}^{+0.020}$ & $1.489_{-0.034}^{+0.036}$ & $1.489_{-0.007}^{+0.036}$ & $1.471_{-0.010}^{+0.069}$ & $10^{10} \mathcal{M}_{\odot}$ \\
\hline
\end{tabular}

error (Table 3). Note that the models presented by Schmidt et al. (1998) and by Chae et al. (1998) fit most badly the D image flux ratio whereas our best models fit most badly the B image flux ratio.

Adding an external shear perturbation (PIE+XS model) we got the best fit up to now: $\chi_{\mathrm{df}}^{2}=0.35$ - the positions are fitted within their errors which are small enought to say that the positions are fitted well. Note that the flux ratios are fitted almost with the same accuracy as in the SIE model, and that the low $\chi^{2}$ comes from the accurate fitting of the positions (Table 3 ). Our result confirms the finding of Keeton et al. (1997) that the independent source of shear is more fundamental variable than the changes in the radial mass distribution (see also Schmidt et al. 1998). Furhermore, Witt \& Mao (1997) using an analytical approach found the minimal shear required to fit the position to be quite small $-0.0099 \pm 0.0063$. Our numerical result for the shear magnitude $\left(\gamma=0.012_{-0.001}^{+0.005}\right)$ is consistent with this lower limit but slightly larger than it (we try to fit the positions and the magnifications simultaneously).

We made some further experiments with the SIE, the PIE and the PIE+XS models (in Table 4 we show the results from the experiments for the PIE $+\mathrm{XS}$ model). Firstly, we minimized these models removing the constraints that come from the flux ratios. The PIE $+\mathrm{XS}$ model was run for several different model velocity dispersions while minimizing over the remaining parameters
Table 3. The contribution of the position and flux ratio terms $\left(\chi_{\theta, i}^{2}, \chi_{\mu, i}^{2}\right)$ to the final $\chi_{\min }^{2}$ for each image and for each model

\begin{tabular}{lcccr}
\hline Model & B image & C image & D image & $\chi_{\min }^{2}$ \\
\hline SIE & $73.00,0.43$ & $53.55,0.16$ & $92.00,0.12$ & 219.26 \\
PIE & $10.80,0.72$ & $11.89,0.31$ & $14.68,3.02$ & 41.42 \\
PIE+XS & $0.080,0.52$ & $0.110,0.14$ & $0.040,0.17$ & 1.06 \\
PIE+SIS & $0.010,0.49$ & $0.006,0.15$ & $0.004,0.22$ & 0.88 \\
\hline
\end{tabular}

(the solution for the PIE+XS model is the one with the lowest $\left.\chi_{\mathrm{df}}^{2}\right)$. For all three models we found that the parameters of the lens models were very close to those listed in Table 2 (see Table 4). The corresponding values of $\chi_{\min }^{2} / n_{\mathrm{df}}$ for the SIE, the PIE and the PIE+XS models are: $218.52 / 3,37.33 / 2$ and $0.23 / 1$, respectively (cf. Table 3 ). Secondly, we decreased the errors of the radio flux ratios by a factor of 100 in order the accuracy of the positions and of the ratios to be comparable, i.e. we simulated high accurate flux ratios. Then we minimized the above three models again. We found slightly different parameters compared with those listed in Table 2 (see Table 4) but a very poor fit $-\chi_{\mathrm{df}}^{2} \approx 1000$ for all three models. So, the high accurate flux ratios make our models unacceptable from the $\chi^{2}$ point of view. However, to be sure in this conclusion high accurate observed but not simulated ratios should be used. From these experiments 
Table 4. Parameters of the PIE + XS model for three different sets of constraints: I - observed positions and ratios; II - observed positions; III - observed positions and simulated ratios (the errors of the radio flux ratios were decreased by a factor of 100)

\begin{tabular}{ccccc}
\hline Parameter & I & II & III & Unit \\
\hline$\sigma_{\mathrm{m}}$ & 182.3 & $182.3^{\mathrm{f}}$ & 184.5 & $\mathrm{~km} \mathrm{~s}^{-1}$ \\
$\varpi$ & 0.000 & 0.000 & 0.005 & $\operatorname{arcsec}$ \\
$\varepsilon$ & 0.195 & 0.195 & 0.265 & - \\
$\varphi_{\varepsilon}$ & 154.3 & 154.4 & 156.5 & degree \\
$\gamma$ & 0.012 & 0.012 & 0.010 & - \\
$\varphi_{\gamma}$ & 110.0 & 109.6 & 136.5 & degree \\
\hline
\end{tabular}

$\mathrm{f}$ the parameter was fixed during the minimization (see the text).

we could conclude that with the present accuracy of the flux ratios the model parameters are determined mainly by the constraints coming from the observed image positions. Despite this, we are forced to use the flux ratios in order to ensure enough observational constraints for the $\mathrm{PIE}+\mathrm{XS}$ and for the PIE+SIS models. Furthermore, high accurate flux ratios are needed for the future modelling in order tightly to constrain the range of the acceptable models of the GLS Q 2237+0305 (Agol et al. 2000; see also Chae et al. 1998).

The introduction of the second deflecting mass (at $z=0.5664$; PIE+SIS model) along with the main lens gives an excellent fit too $\left(\chi_{\mathrm{df}}^{2}=0.44\right)$ - the slightly larger $\chi_{\mathrm{df}}^{2}$ compared to the PIE $+\mathrm{XS}$ model is due to the worse fitting of the $\mathrm{C}$ and $\mathrm{D}$ images flux ratio. Note however, that under the present observational constraints the PIE+XS and the PIE+SIS models are formally undistinguishable from the $\chi^{2}$ point of view - both models fit the observations within their observational errors. The parameters of the mass distribution are in very close agreement with those of the PIE+XS model (Table 2), and the direction to the second lens and the direction to the mass excess inferred from the PIE $+\mathrm{XS}$ model are aligned up to $\approx 4^{\circ}$ (Fig. 2; we should point out that the orientation of the shear and the position of the second lens are not well constrained by the models). The mass excess angle is far away from the directions to the galaxy bar and to Pegasus II cluster. These results together with the better fit compared to that of Schmidt et al. (1998) mean that the main source of the shear can be the object connected with the MgII absorption and not the bar, nor the mass of Pegasus II cluster. In any case one must include perturbations (either expanding or explicitly taking into account the perturbers' potentials) in order to get a satisfactory fit to the GLS Q 2237+0305 observations.

\subsection{Mass distribution in the main lens $(z=0.0394)$}

Our discussion on the parameters of the SBb galaxy mass distribution will start with the core radius. The PIE model has a core radius of $260 \pm 16 \mathrm{pc}$ that is close to the approximate upper limit of 200 pc inferred from a statistical study by Wallington \& Narayan (1993). Note that the calculated position of the fifth quasar image is in good agreement with Racine's (1991) position of the probable fifth image and that the calculated flux ratio $\mu_{\mathrm{EA}}^{\text {calc }}$ is below the upper limit set by Falco et al. (1996). If the feature observed by Racine (1991) is indeed the fifth quasar image then a strong constraint on the core radius will be imposed. The problem is the poor fit obtained by the PIE model. Taking into account the lensing effect of the external perturbations or the effect of the second lens we improve the fit and get the mass profiles that are consistent with the singular profile. Accounting for the PIE+SIS model we can set an approximate upper limit on the core radius of $0 . \prime 05(=36 \mathrm{pc})$. The core radius of $40 \mathrm{pc}$ found by Chae et al. (1998) is consistent with this limit. Schmidt et al. (1998) achieved a good fit to the observations with a singular nearly pseudoisothermal elliptical mass distribution plus a Ferrers bar acting as a perturber. These results point toward a mass model for the GLS Q 2237+0305 that is nearly singular at the centre.

The standard models tightly constrain the position angle of the model major axis - averaging over all presented up to now standard models we get $\mathrm{PA}=66^{\circ} .8 \pm 0.5$. Fitte \& Adam (1994) claim the average position angle of the major axis of the light distribution inside $\theta=1^{\prime \prime} .25$ to be $77^{\circ}$ which leads to about $10^{\circ}$ misalignment between the light and the mass distribution. This misalignment could be a real property of the lensing SBb galaxy (e.g. Keeton et al. 1997) or the model major axis could represent the resulting shear orientation if there are lensing components that have not been included in the standard models (Schmidt et al. 1998). Accounting for the relatively good fit obtained by Schmidt et al. (1998), we shall furthermore consider only the second possibility. Our best models increased this misalignment up to $\approx 13^{\circ}$ toward the bar. This misalignment between the model mass distribution and the bulge+disk light distribution means that the bar has non-neglegible lensing effect (Schmidt et al. 1998) and should be included in the detailed modelling of the GLS Q 2237+0305.

From our best models we get the mean axis ratio to be 0.676 which is consistent with the result of 0.7 derived by Fitte \& Adam (1994) for the central part of the SBb galaxy.

Foltz et al. (1992) measured the central velocity dispersion $\sigma_{\mathrm{c}}$ of the lensing SBb galaxy to be $215 \pm 30 \mathrm{~km} \mathrm{~s}^{-1}$. Rix et al. (1992) reanalyzed their data and found the central velocity dispersion (in a $0 . .7 \times 0.9$ aperture) to be $209 \pm 19 \mathrm{~km} \mathrm{~s}^{-1}$. Using Fig. 6 of Rix et al. (1992) we can make a rough estimate of the central velocity dispersion for a spherical, nonrotating, isotropic, Hernquist (1990) model. Taking the SBb galaxy effective radius of $10^{\prime \prime} 6$ (between $\theta=00^{\prime \prime} .25$ and $\theta=2$ '. 1 , Fitte \& Adam 1994) we obtain $\sigma_{\mathrm{c}} \approx 184 \mathrm{~km} \mathrm{~s}^{-1}$. On the other hand, Kochanek (1993) proved that the average velocity dispersion $\sigma_{\mathrm{c}}$ within the central few arcseconds is roughly 


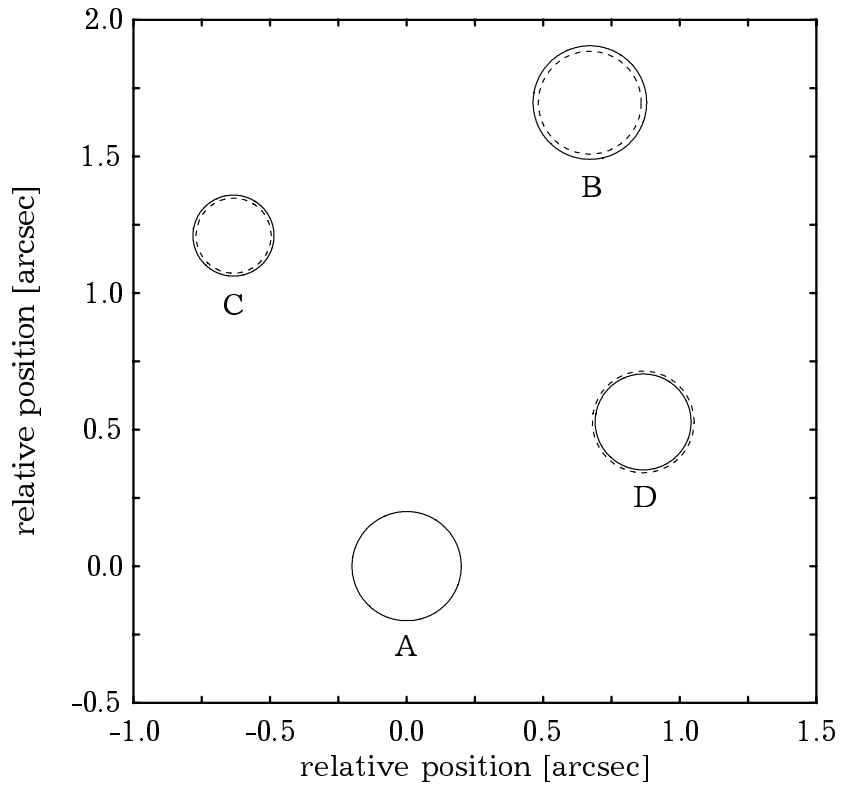

Fig. 1. The observed (solid circles) and the predicted (dashed circles) by the PIE+XS model image positions and ratios. The flux ratio $\mu_{i \mathrm{~A}}$ is equal to the ratio of the area of the $i$ th image circle to the area of the A image circle

equal to the model velocity dispersion $\sigma_{\mathrm{m}}$ (see also Breimer \& Sanders 1993). We straightforward assume that $\sigma_{\mathrm{c}}=\sigma_{\mathrm{m}}$ for an isotropic, spherically symmetric model, i.e. our best models predict $\sigma_{\mathrm{c}}=181.7 \mathrm{~km} \mathrm{~s}^{-1}$ (this value is an average over the PIE+XS and the PIE+SIS models). Therefore, our estimate of the central velocity dispersion based on the lens modelling is in agreement with the dynamical estimate for an isotropic spherical model. If one breaks the spherical symmetry, the velocity dispersion will be reduced by a factor $\left(\sqrt{1-e^{2}} \sin ^{-1} e\right) / e$, where the eccentricity is $e=\sqrt{1-f_{0}^{2}}$ and $f_{0}$ is the edge-on axis ratio (Kent \& Falco 1988). Chae et al. (1998) obtained $f_{0}=0.835$ and therefore, the central velocity dispersion predicted by our models is $\sigma_{\mathrm{c}}=160.7 \mathrm{~km} \mathrm{~s}^{-1}$. This value is in agreement with the results of Kent \& Falco (1988) who used different radial mass profiles for the SBb galaxy mass model. Our value of the central velocity dispersion (for an isotropic model) is smaller than the measured value of $209 \mathrm{~km} \mathrm{~s}^{-1}$. This points to the presence of some degree of anisotropy in the central part of the lensing galaxy and that the radial component of the velocity dispersion is greater than the tangential one (Foltz et al. 1992; see also Fig. 1 of Richstone \& Tremaine 1984). Note that, if there is only slight anisotropy in the galaxy centre, corrections up to $40 \%$ in the isotropic central velocity dispersion are possible (Tonry 1983; see also Foltz et al. 1992), i.e. we obtain $\sigma_{\mathrm{c}}=160.7 \pm 64.3 \mathrm{~km} \mathrm{~s}^{-1}$ from the lens modelling which result agrees well at its upper limit with the observations.

Huchra et al. (1985) made a crude estimate of the rotational velocity at the position $5^{\prime \prime}$ to the West of the $\mathrm{SBb}$ galaxiy nucleus: $v_{\text {rot }}=260 \pm 75 \mathrm{~km} \mathrm{~s}^{-1}$. The mean rotational velocity predicted by our PIE $+\mathrm{XS}$ and PIE+SIS

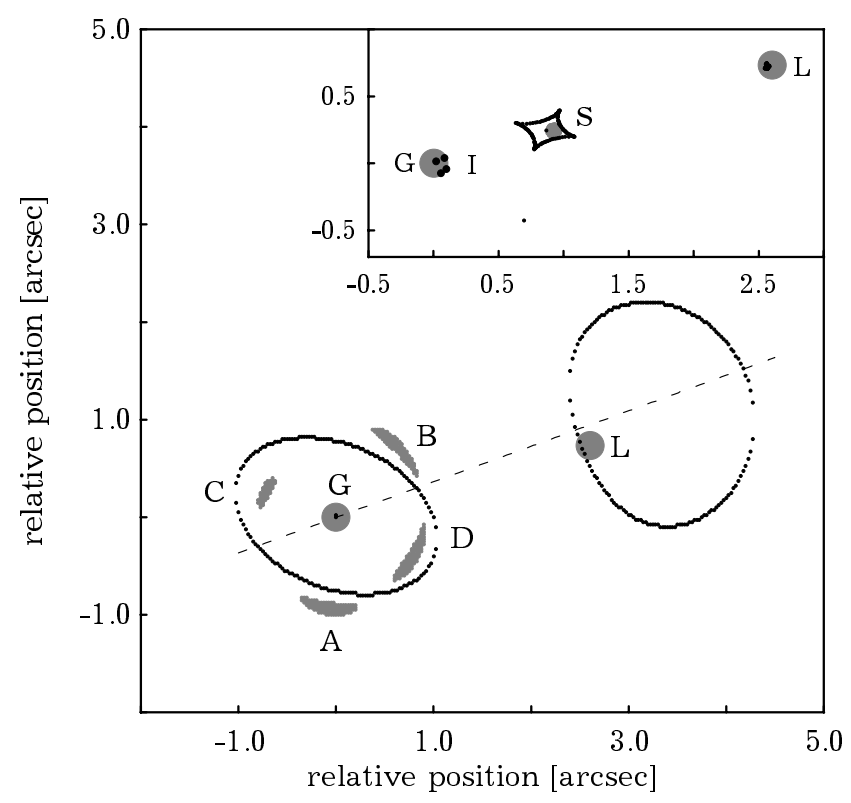

Fig. 2. Critical curves for the PIE+SIS model. The images (grey spots), the SBb galaxy (grey circle labelled $\mathrm{G}$ ) and the second lens (grey circle labelled L) are shown. The insert shows the caustics in the source plane together with the source (grey circle labelled S), the lenses (shown as grey circles), and the points where the images intersect the second plane (four points marked I). The dashed line represents the direction to the mass excess found by the PIE+XS model

models at a distance of $5^{\prime \prime}$ is about $257 \mathrm{~km} \mathrm{~s}^{-1}$ which is within the error of the measured value. The asymptotic (at $\theta \gg \varpi$ ) rotation velocity is $\sqrt{2} \sigma_{\mathrm{m}}$ or about $257 \mathrm{~km} \mathrm{~s}^{-1}$ which is the same as above because of the vanishing core size.

The introduction of the second lens plane decreases the mass inside the mean ring of the images with $1.2 \%$ relative to the one plane models (Table 2; we adopt $\left.\mathcal{M}_{\odot}=1.98910^{33} \mathrm{~g}\right)$. Schmidt et al. (1998) obtained the mass inside the circle of 0.9 radius $\mathcal{M}\left(<0^{\prime \prime} .9\right)=(1.49 \pm$ 0.01) $10^{10} \mathcal{M}_{\odot}$. For the same radius the PIE+XS and the PIE+SIS models give $\mathcal{M}\left(<0^{\prime \prime} 9\right)=1.514_{-0.007}^{+0.036} 10^{10} \mathcal{M}_{\odot}$ and $\mathcal{M}(<0$ '. 9$)=1.495_{-0.009}^{+0.071} 10^{10} \mathcal{M}_{\odot}$, respectively. One can see that the mass obtained by our PIE+SIS model is in excellent agreement with the results of Schmidt et al. (1998) who used a different lens model. For the circle of radius $1^{\prime \prime}$ the PIE $+\mathrm{XS}$ and the PIE+SIS models give $\mathcal{M}\left(<1^{\prime \prime}\right)=1.682_{-0.007}^{+0.041} 10^{10} \mathcal{M}_{\odot}$ and $\mathcal{M}\left(<1^{\prime \prime}\right)=$ $1.662_{-0.011}^{+0.078} 10^{10} \mathcal{M}_{\odot}$, respectively. The $r$ magnitude of the $\mathrm{SBb}$ galaxy inside the same radius is $r\left(<1^{\prime \prime}\right)=16.75$ (cited in Foltz et al. 1992). Using the galaxy colour $g-r=0.48$ (Yee 1988) and the transformation equation $B=0.437+r+1.589(g-r)$ (Corrigan et al. 1991) we get the $B$ magnitude $17^{\mathrm{m}} 95$. Adopting the absolute $B$ magnitude of the Sun to be $M_{\odot, B}=5$. 41 we obtain the blue mass-to-light ratio for the central $1^{\prime \prime}$ to be $\mathcal{M} / \mathcal{L}_{B}=$ $6.9_{-0.1}^{+0.2} \mathcal{M}_{\odot} / \mathcal{L}_{\odot, B}$ and $\mathcal{M} / \mathcal{L}_{B}=6.8_{-0.1}^{+0.3} \mathcal{M}_{\odot} / \mathcal{L}_{\odot, B}$ for the PIE+XS and the PIE+SIS models, respectively. For the inner $10^{\prime \prime}$ of the galaxy Rix et al. (1992) found 
$\mathcal{M} / \mathcal{L}_{B}=(9.2 \pm 1.5) \mathcal{M}_{\odot} / \mathcal{L}_{\odot, B}$ having used a constant mass-to-light ratio model for the SBb galaxy. Our estimates are in rough agreement with this result as well as with the results obtained for the spiral galaxies (e.g. Rubin et al. 1985). Finally, there will be additional uncertainity in the two-plane mass estimation due to the mass at $z \approx 0.97$.

\subsection{Mass distribution in the second lens $(z=0.5664)$}

For the present, the most popular hypothesis for the origin of the Mg II absorption lines in the quasars' spectra is that the absorption arises in the gaseous halos of the field galaxies whose halos intersect the quasars' line-of-sight (Bergeron \& Boissé 1991; Bergeron et al. 1992; Bechtold \& Ellingson 1992; Le Brun et al. 1993). Under this hypothesis we assume that the object associated with the Mg II absorption is a single galaxy. Following the discussion in Sect. 3.2 we obtain the central velocity dispersion of the second galaxy-lens $\sigma_{\mathrm{c}}^{(2)} \lesssim 253.6_{-80.4}^{+29.2} \mathrm{~km} \mathrm{~s}^{-1}$ with possible additional uncertainity up to $40 \%$. This central velocity dispersion is somewhat higher for a spiral galaxy (see Whitmore et al. 1985), so, we assume that the second lens is an elliptical galaxy (note however, that the model velocity dispersion of the second lens is not well constrained by the model, so, we cannot reject the possibility the second lens to be a spiral galaxy at the lower end of the confidence interval). Using Faber-Jackson relationship $\mathcal{L}=\mathcal{L}_{*}\left(\sigma_{\mathrm{c}} / \sigma_{\mathrm{c} *}\right)^{a}$ (Faber \& Jackson 1976) with paprameters $\sigma_{\mathrm{c} *}=225 \mathrm{~km} \mathrm{~s}^{-1}$ and $a=3.6$ for ellipticals (de Vaucouleurs \& Olson 1982) we get the luminousity of the second lensing galaxy $\mathcal{L}^{(2)} \approx 1.6 \mathcal{L}_{*}$. The mean projected angular distance between the second lens and the points where the quasar images intersect the second plane is 2 '.647 (=13.2 kpc). This sets a lower limit on the radius of the gaseous halo (where the Mg II absorption arises) associated with the second lens of $\approx 14 \mathrm{kpc}$. Hewett et al. (1994) obtained the lower limit on the radius of the Mg II absorber of $\approx 27 \mathrm{kpc}$ based on the observations of the gravitationally lensed system Q 1009-0252. From an imaging study Bergeron \& Boissé (1991) found the gaseous halos to extend up to $\approx 50 \mathrm{kpc}$ which is consistent with the results obtained from lensing. Finally, being given only the velocity dispersion of the absorber we cannot reject the possibility the object associated with the Mg II absorption to be a small group of galaxies (Yanny \& York 1992; Drinkwater et al. 1993).

The introduction of the second lens plane weakens the statistical difficulties connected with this system (e.g. Schneider et al. 1988) - there is no need of close alignment between the source and the main lens (the impact parameter, i.e. the separation between the source and the $\mathrm{SBb}$ galaxy, is $0^{\prime \prime} 062$ for the PIE+XS model compared with $0^{\prime \prime} 956$ for the PIE+SIS one). On the other hand, we need two lenses at a different redshift to be aligned better than $3^{\prime \prime}$. Following Kochanek \& Apostolakis (1988) we derive the probability to find a second lens 2 .'704 away from the main $\mathrm{SBb}$ lens to be $8.310^{-5}$. The self-consistent probability (see Ehlers \& Schneider 1986) of getting the five image system is $9.610^{-5}$. To derive the above probabilities we have used a number density of spiral lenses $n(z)=n(0)(1+z)^{3}$, where $n(0)=1.510^{-2} h^{3} \mathrm{Mpc}^{-3}$ (Marzke et al. 1994). Therefore, the total probability for the PIE+SIS model is $8.010^{-9}$. This is a very low probability; the existence and the discovery of the GLS Q $2237+0305$ should be considered as a statistical fluke (see also Kochanek 1992). The low probability for the PIE+SIS model means that it is unlikely for another lensed system as GLS Q 2237+0305 to exist among the nearby galaxies - the GLS Q 2237+0305 remains a unique gravitationally lensed system, an exception among the lensed systems.

For comparison, for the GLS Q $2345+007$ the probability for the two-plane lens is estimated to be $\approx 1.210^{-3}$ or less depending on the solution type adopted (Subramanian \& Chitre 1984). However, the GLS Q $2345+007$ is a two image system and the lensing galaxy is a high redshift galaxy ( $z \approx 1.5$, Fischer et al. 1994), so one gets higher probability compared with the GLS Q $2237+0305$.

\section{Conclusions}

In this paper we have presented one-plane lens models (with and without an external shear added) and a twoplane lens model for the GLS Q 2237+0305. The first (the main) lens plane is at $z=0.0394$ and the second lens plane is at $z=0.5664$. All models are based on an isothermal mass distribution (Kassiola \& Kovner 1993).

We summarize the findings of this paper as follows:

1. We modelled the GLS Q 2237+0305 using the most accurate image positions (HST) up to now and realistic radio flux ratios (VLA) both used as observational constraints;

2. We found the best gravitational lens solutions for the GLS Q $2237+0305$ up to now $-\chi_{\text {df }}^{2}$ equals to 0.35 and 0.44 for the PIE $+\mathrm{XS}$ and the PIE + SIS models, respectively, showing that the main source of the external shear in this system is probably the object associated with the $\mathrm{Mg}$ II absorption at $z=0.5664$;

3 . We derived the parameters of the pseudoisothermal mass distribution (averaged over the $\mathrm{PIE}+\mathrm{XS}$ and the PIE+SIS models unless otherwise specified) in the central part of the lensing SBb galaxy: a model velocity dispersion $\sigma_{\mathrm{m}}=181.7 \mathrm{~km} \mathrm{~s}^{-1}$ which translates to a central velocity dispersion $\sigma_{\mathrm{c}}=160.7 \pm$ $64.3 \mathrm{~km} \mathrm{~s}^{-1}$ and to an asymptotic rotational velocity $v_{\text {rot }} \approx 257 \mathrm{~km} \mathrm{~s}^{-1}$; an upper limit on the core radius of 36 pc (PIE+SIS model); a minor-to-major axis ratio $f=0.676$; a position angle of the model major axis $\mathrm{PA}=64.3$;

4. The introduction of the second lens plane leaves the core radius, the axis ratio and the position angle of the mass distribution in the main lens almost unchanged but decreases the mass inside the mean ring of the 
images with $1.2 \%$ and increases the total magnification of the images by a factor of $\approx 1.5$;

5. For the PIE+SIS model the mass of the SBb galaxy inside the mean ring of the images is $\mathcal{M}(\leqq 0$ '! 885) $=$ $1.471_{-0.010}^{+0.069} 10^{10} \mathcal{M}_{\odot}$ and the blue mass-to-light ratio for the central $1^{\prime \prime}$ of the $\mathrm{SBb}$ galaxy is $\mathcal{M} / \mathcal{L}_{B}=$ $6.8_{-0.1}^{+0.3} \mathcal{M}_{\odot} / \mathcal{L}_{\odot, B}$

6 . We determined some parameters of the second lens which is connected with the MgII absorption (assuming that the second lens is a single elliptical galaxy) as follows: a model velocity dispersion $\sigma_{\mathrm{m}}^{(2)}=$ $253.6_{-80.4}^{+29.2} \mathrm{~km} \mathrm{~s}^{-1}$ which translates to a central velocity dispersion $\sigma_{\mathrm{c}}^{(2)} \lesssim 253.6_{-80.4}^{+29.2} \mathrm{~km} \mathrm{~s}^{-1}$ with possible additional uncertainity up to $40 \%$; a luminousity $\mathcal{L}^{(2)} \approx$ $1.6 \mathcal{L}_{*}$; radius of the $\mathrm{Mg}$ II absorbing halo greater than $\approx 14 \mathrm{kpc}$

7. The probability for the two-plane lens model PIE+SIS is estimated to be $8.010^{-9}$.

8. We found the $B$ magnitudes for the unlensed quasar to be $19^{\mathrm{m}} 69$ and $20^{\mathrm{m}} 15$ for the PIE $+\mathrm{XS}$ and the PIE+SIS models, respectively.

Accounting for the presented models as well as for the models of Schmidt et al. (1998) and of Chae et al. (1998) we can claim that the mass distribution in the central part of the $\mathrm{SBb}$ galaxy is nearly singular and nearly isothermal and that the contribution of the bar and of the $\mathrm{Mg}$ II absorbers to the lensing must be taken into account.

To go further in the study of the mass distribution in the GLS G 2237+0305 via gravitational lensing we need more accurate observational constraints. These could include: HST observations from IR to UV bands in order to get accurate image and $\mathrm{SBb}$ galaxy positions as well as to derive accurately the light distribution in the central part of the SBb galaxy (where most of the mass relevant for the lensing is situated); emission line imaging in order to get constraints from the observed arc of extended emission; radio and infrared observations in order to determine accurately the flux ratios; spectral observations in order to determine accurately the velocity dispersion and the rotation curve of the $\mathrm{SBb}$ galaxy. Deep optical, infrared and radio observations could also set a tight upper limit on the flux ratio of the fifth quasar image (or even detect it!). Furthermore, we have to explore more complicated lens mass models - any final model must include perturbations (we have either to expand or explicitly to take into account the perturbers' potentials).

It will be interesting to obtain a mass estimation for the $\mathrm{SBb}$ galaxy-lens via other methods - analysis of the rotation curve and of the light distribution, and to compare the mass obtained through that analysis with its lensing mass. The proximity of the main lens in the GLS Q $2237+0305$ provides for us this opportunity.

Acknowledgements. The author is thankful to Dr. G. Petrov and Dr. A. Strigachev for useful discussions and to $\mathrm{PhD}$ student L. Slavcheva-Mihova for the help in preparing the manuscript. Special thanks to the anonymous referee whose critical comments significantly improved the manuscript.

\section{References}

Agol, E., Jones, B., \& Blaes, O. 2000, ApJ, 545, 657

Bechtold, J., \& Ellingson, E. 1992, ApJ, 396, 20

Bergeron, J., \& Boissé, P. 1991, A\&A, 243, 344

Bergeron, J., Cristiani, S., \& Shaver, P. A. 1992, A\&A, 257, 417

Blandford, R. D., \& Narayan, R. 1992, ARA\&A, 30, 311

Blanton, M., Turner, E. L., \& Wambsganss, J. 1998, MNRAS, 298, 1223

Breimer, T. G., \& Sanders, R. H. 1993, A\&A, 274, 96

Burud, I., Stabell, R., Magain, P., et al. 1998, A\&A, 339, 701

Chae, K.-H., Turnshek, D. A., \& Khersonsky, V. K. 1998, ApJ, 495,609

Chincarini, G., \& Rood, H. J. 1976, PASP, 88, 388

Corrigan, R. T., Irwin, M. J., Arnaud, J., et al. 1991, AJ, 102, 34

Crane, P., Albrecht, R., Barbieri, C., et al. 1991, ApJ, 369, L59

de Vaucouleurs, G., \& Olson, D. W. 1982, ApJ, 256, 346

Drinkwater, M. J., Webster, R. L., \& Thomas, P. A. 1993, AJ, 106,848

Ehlers, J., \& Schneider, P. 1986, A\&A, 168, 57

Erdl, H., \& Schneider, P. 1993, A\&A, 268, 453

Faber, S. M., \& Jackson, R. E. 1976, ApJ, 204, 668

Falco, E. E., Lehár, J., Perley, R. A., Wambsganss, J., \& Gorenstein, M. V. 1996, AJ, 112, 897

Fischer, P., Tyson, J. A., Bernstein, G. M., \& Guhathakurta, P. 1994, ApJ, 431, L71

Fitte, C., \& Adam, G. 1994, A\&A, 282, 11

Foltz, C. B., Hewett, P. C., Webster, R. L., \& Lewis, G. F. 1992, ApJ, 386, L43

Hernquist, L. 1990, ApJ, 356, 359

Hewett, P. C., Irwin, M. J., Foltz, C. B., et al. 1994, AJ, 108, 1534

Hintzen, P., Maran, S. P., Michalitsianos, A. G., et al. 1990, AJ, 99, 45

Huchra, J., Gorenstein, M., Kent, S., et al. 1985, AJ, 90, 691

Irwin, M. J., Webster, R. L., Hewett, P. C., Corrigan, R. T., \& Jedrzejewski, R. I. 1989, AJ, 98, 1989

Kayser, R., \& Schramm, T. 1988, A\&A, 191, 39

Kayser, R. 1990, ApJ, 357, 309

Kassiola, A., \& Kovner, I. 1993, ApJ, 417, 450

Kassiola, A., \& Kovner, I. 1995, MNRAS, 272, 363

Keeton, C. R., Kochanek, C. S., \& Seljak, U. 1997, ApJ, 482, 604

Keeton, C. R., Kochanek, C. S., \& Falco, E. E. 1998, ApJ, 509, 561

Keeton, C. R., Mao, S., \& Witt, H. J. 2000, ApJ, 537, 697

Kent, S. M., \& Falco, E. E. 1988, AJ, 96, 1570

Kochanek, C. S., \& Apostolakis, J. 1988, MNRAS, 235, 1073

Kochanek, C. S. 1991, ApJ, 373, 354

Kochanek, C. S. 1992, ApJ, 397, 381

Kochanek, C. S. 1993, ApJ, 419, 12

Le Brun, V., Bergeron, J., Boissé, P., \& Christian, C. 1993, A\&A, 279, 33

Marzke, R. O., Geller, M. J., Huchra, J. P., \& Corwin, Jr. H. G. 1994, AJ, 108, 437

Mediavilla, E., Arribas, S., del Burgo, C., et al. 1998, ApJ, 503, L27

Minakov, A. A., \& Shalyapin, V. N. 1991, AZh Lett., 17, 331

Østensen, R., Refsdal, S., Stabell, R., et al. 1996, A\&A, 309, 59

Petrov, G., Strigachev, A., \& Mihov, B. 1996, C.r. l'Acad. bulg. Sci., 49,5 
Press, W. H., Flannery, B. P., Teukolski, S. A., \& Vetterling, W. T. 1986, Numerical Recipes (Cambridge Univ. Press, Cambridge)

Racine, R. 1991, AJ, 102, 454

Richstone, D. O., \& Tremaine, S. 1984, ApJ, 286, 27

Rix, H.-W., Schneider, D. P., \& Bahcall, J. N. 1992, AJ, 104, 959

Rubin, V. C., Burstein, D., Kent Ford, Jr. W., \& Thonnard, N. 1985, ApJ, 289, 81

Schmidt, R., Webster, R. L., \& Lewis, G. F. 1998, MNRAS, 295,488

Schneider, D. P., Turner, E. L., Gunn, J. E., et al. 1988, AJ, 95, 1619

Schramm, T., \& Kayser, R. 1987, A\&A, 174, 361

Subramanian, K., \& Chitre, S. M. 1984, ApJ, 276, 440

Teuber, J., Østensen, R., Stabell, R., \& Florentin-Nilsen, R. 1994, A\&AS, 108, 509

Tonry, J. L. 1983, ApJ, 266, 58

Trotter, C. S., Winn, J. N., \& Hewitt, J. N. 2000, ApJ, 535, 671
Vakulik, V. G., Dudinov, V. N., Zheleznyak, A. P., et al. 1997, Astron. Nachr., 318, 73

Wallington, S., \& Narayan, R. 1993, ApJ, 403, 517

Wambsganss, J., \& Paczyński, B. 1994, AJ, 108, 1156

Webster, R. L., Ferguson, A. M. N., Corrigan, R. T., \& Irwin, M. J. 1991, AJ, 102, 1939

Williams, L. L. R., \& Saha, P. 1995, AJ, 110, 1471

Whitmore, B. C., McElroy, D. B., \& Tonry, J. L. 1985, ApJS, 59,1

Witt, H. J., Mao, S., \& Schechter, P. L. 1995, ApJ, 443, 18

Witt, H. J. 1996, ApJ, 472, L1

Witt, H. J., \& Mao, S. 1997, MNRAS, 291, 211

Yanny, B., \& York, D. G. 1992, ApJ, 391, 569

Yee, H. K. C. 1988, AJ, 95, 1331

Zwicky, F., Karpowicz, M., \& Kowal, C. 1965, Catalogue of Galaxies and Clusters of Galaxies (California Institute of Technology, Pasadena), vol. V 